\title{
Erratum
}

\section{Three-fluid plasmas in star formation}

\section{Momentum transfer rate coefficients}

\author{
C. Pinto ${ }^{1}$ and D. Galli ${ }^{2}$ \\ 1 Dipartimento di Astronomia e Scienza dello Spazio, Università di Firenze, Largo E. Fermi 5, 50125 Firenze, Italy \\ 2 INAF - Osservatorio Astrofisico di Arcetri, Largo E. Fermi 5, 50125 Firenze, Italy
}

A\&A 484, 17-28 (2008), DOI: 10.1051/0004-6361:20078819

Key words. atomic processes - molecular processes - plasmas - magnetohydrodynamics (MHD) - ISM: clouds ISM: jets and outflows - errata, addenda

The third line of Table 2 of the paper "Three-fluid plasmas in star formation II. Momentum transfer rate coefficients" by Pinto and Galli, published in Astronomy \& Astrophysics 484, 17-28, contains an error. The correct line is given in Table 2 here. We would like to thank Despina Panoglou and Sylvie Cabrit for finding this error and drawing our attention to it.

Table 2. Fitting formulae for momentum transfer coefficients as function of $v_{\mathrm{rms}}$ (in $\mathrm{km} \mathrm{s}^{-1}$ ).

\begin{tabular}{|c|c|c|}
\hline Species $s, s^{\prime}$ & $\begin{array}{l}\langle\sigma v\rangle_{s s^{\prime}} \\
\left(\mathrm{cm}^{3} \mathrm{~s}^{-1}\right)\end{array}$ & $\begin{array}{l}v_{\mathrm{rms}} \\
\left(\mathrm{km} \mathrm{s}^{-1}\right)\end{array}$ \\
\hline $\mathrm{HCO}^{+}, \mathrm{H}_{2}$ & $2.40 \times 10^{-9} v_{\mathrm{rms}}^{0.6}$ & $0.2 \lesssim v_{\mathrm{rms}} \lesssim 5$ \\
\hline $\mathrm{H}_{3}^{+}, \mathrm{H}_{2}$ & $2.00 \times 10^{-9} v_{\mathrm{rms}}^{0.15}$ & $1 \lesssim v_{\mathrm{rms}} \lesssim 10$ \\
\hline $\mathrm{H}^{+}, \mathrm{H}_{2}$ & $1.35 \times 10^{-9} v_{\mathrm{rms}}^{0.22}$ & $1 \lesssim v_{\mathrm{rms}} \lesssim 10$ \\
\hline$e, \mathrm{H}_{2}$ & $3.16 \times 10^{-11} v_{\mathrm{rms}}^{1.3}$ & $20 \lesssim v_{\text {rms }} \lesssim 200$ \\
\hline $\mathrm{C}^{+}, \mathrm{H}$ & $1.74 \times 10^{-9} v_{\mathrm{rms}}^{0.2}$ & $2 \lesssim v_{\mathrm{rms}} \lesssim 20$ \\
\hline $\mathrm{H}^{+}, \mathrm{H}$ & $2.13 \times 10^{-9} v_{\mathrm{rms}}^{0.75}$ & $v_{\mathrm{rms}} \gtrsim 1(\mathrm{GKS})$ \\
\hline$e, \mathrm{H}$ & $2.50 \times 10^{-10} v_{\mathrm{rms}}^{1.2} \exp \left(-v_{\mathrm{rms}} / 460\right)$ & $20 \lesssim v_{\mathrm{rms}} \lesssim 600$ \\
\hline $\mathrm{H}^{+}, \mathrm{He}$ & $1.48 \times 10^{-9} v_{\mathrm{rms}}^{-0.02}$ & $0.1 \lesssim v_{\mathrm{rms}} \lesssim 10$ \\
\hline$e, \mathrm{He}$ & $7.08 \times 10^{-11} v_{\mathrm{rms}}$ & $20 \lesssim v_{\mathrm{rms}} \lesssim 500$ \\
\hline
\end{tabular}

